\title{
THEMA
}

théorie économique,

modélisation et applications

THEMA Working Paper $\mathrm{n}^{\circ} 2021-01$ CY Cergy Paris Université, France

\section{The Public Good spatial power index in political games}

Arnold Cédrick SOH VOUTSA 


\title{
The Public Good spatial Power INDEX IN POLITICAL GAMES
}

\author{
Arnold Cedrick SOH VOUTSA \\ THEMA, CY Cergy Paris Université. \\ cedricksoh@gmail.com
}

January 6, 2021

\begin{abstract}
We propose a new spatial index called the Public Good spatial index, which is the spatial version of the standard Public Good index under independence behaviors (PGI). However, we show that the spatial model used, is not well suited for both, the standard Shapley Shubik index and the standard Public Good index under Homogeneity behaviors (PGH); and consequently they do not have a consistent spatial version with respect to our model. By contrast, the same spatial model is appropriated for the Banzhaf index and the aforementioned PGI index, concomitantly, allowing their spatial versions in political games. We also argue that those two well convenient spatial indices are only understandable under behavioral descriptions with independence assumptions on political issues. Finally, the paper details our findings by means of examples, comparisons, and it also provides relevant ways of computing spatial power indices in real case studies when it comes to lower dimensions.
\end{abstract}

Key words: political games, spatial power indices, Public Good index, Banzhaf index. Jel classification: C71, D71.

"A common objection, directed at a priori voting power of any kind, is that it is useless because it makes unrealistic assumptions about voters' behavior and the decision-making process and ignores information about their actual behavior." $\leadsto$ Dan S. Felsenthal and Moshé Machover, The measurement of a priori voting power. 


\section{Introduction}

Because of the growing interest in applications of spatial power indices, it is not surprising that there is an extensive discussion of various spatial power indices going on in the literature. We can observe a renaissance of the probabilistic interpretation about spatial power indices, based on political games. This concept was first introduced by Owen (1971) in extending the Shapley-Shubik power index in political games, then followed by Shapley (1977) which gives another technique and viewpoint on it, and the result was thereby known in the literature as the Owen-Shapley index. A few years later, Shenoy (1982) introduced, inspired by the approach of Shapley, the Banzhaf power index for political games, but this time based on the "question of individual outcomes" underlined by Straffin (1977). To the best of our knowledge, the last extension on political games about well-known classic power indices, was the Deegan-Packel index proposed by Rapoport and Golan (1985). Since then, it appeared in the literature, the formulation of new approaches of spatial indices, and more recently, a series of real applications (Brams et al., 1983; Passarelli and Barr. 2007; Barr and Passarelli, 2009; Alonso-Meijide et al., 2011; Benati and Marzetti, 2013. Mielcová, 2016; Grech, 2020, etc.). To date, no spatial version of the Public Good power index has been published, and this paper attempt to fulfill this gap in political games. Our method is in many ways similar to that of Shenoy, but since the Straffin's question of outcome previously mentioned is not appropriated for the Public Good index, we need to take under consideration another probabilistic interpretation of the Public Good index,

which is provided along with several interpretations in Brueckner (2001). We thus infer from those probabilistic interpretations that, only one of them is well suited for the spatial model considered throughout this work - that interpretation, as we will see in the present paper, is the one based on the independence assumption of voting behaviors.

In the global literature on power indices, a popular view is that a power index represents a reasonable expectation of a collective decision-making outcome. If the collective decision- 
making can be described by a model, then a power index should answer the question: what is the probability that a voter $i$ is decisive for the collective outcome, given that we have no other information on the voting, the voters' preferences and the formation of coalitions? At this level of abstraction, the notion of power index represents an a priori evaluation of the voting game to each player. However, combine empirical results with adequate theories about coalition formation and voting behavior, may improve the probability estimates which we get from the abstract power indices. There are oftentimes called empirical power indices, and they are only used when such empirical results are available. In this work they are applied on spatial voting games (well-suited for political games). We will then use terminologies like spatial power indices to precise the link with our spatial model; also our analysis of spatial power indices is deeply related to the analysis of coalition formation. Lastly, as the ongoing groundwork indicates that the well-established Owen-Shapley spatial index suffers by the so called "too much concentration of power" as argued by many authors like (Passarelli and Barr, 2007, Benati and Marzetti, 2013, among others), in our model, we do put in light that such a concentration's problem does not occur. Furthermore, many improvements and differences between our model to those which already exist in the literature, are highlighted at length.

Our contribution. The development of power indices over the past five decades has brought many new insights into the spatial voting theory. Surprisingly, the insight proposed by Shenoy (1982) about a Banzhaf spatial index has gone almost untouched by game theory researchers. To date, no one has published any formal analyze of that index. This article redresses this neglect and besides, from the result of an 1990 article of Leech (1990) published in Public Choice which weakened the assumptions proposed by Straffin (1977), we deeply investigate the work of Shenoy (1982) and uptate his result. Furthermore, by considering the results of Brueckner (2001), we have pushed forward that investigation and as a result propose a new spatial power index — the Public Good spatial index. Therefore, 
since Shenoy (1982) proposed his model, this paper provides the first formal analysis of his work whereby, we infer the first spatial version of the Public Good index. Lastly, in addition to detail how the model works in practice, our paper also presents examples and comparisons.

Organization of the paper. Preliminaries on simple games and probabilistic interpretations of power indices are presented in the next section. Section 3 introduces the spatial model and the main spatial power indices of the paper, among others. Section 4 provides many illustrations, examples and comparisons. Lastly, section 5 is devoted to concluding remarks.

\section{Simple games}

\subsection{Notation and definitions}

Throughout this document, we will use the following basic notation. Let $N=\{1,2, \ldots, n\}$ denote the set of all players indexed by the first $n$ natural numbers. Any nonempty subset of $N$ is called coalition. Let $2^{N}$ denote the set of all possible coalitions. All $n$ - person cooperative games that model voting bodies in which each coalition can either "win" or "lose" are called simple games (Von Neumann and Morgenstern, 1947). In other words, simple games form a certain class of n-person cooperative games in which each coalition is either all powerful or completely ineffectual. First, we denoted by $\mathcal{G}_{N}$ the set of all simple games with the player set $N$, where a simple game on $N$ is defined as follows.

Definition 1 A simple game consists of a finite set of players represented by $N$, and a collection $\mathcal{W}$ of subsets of $N$ satisfying the following properties:

- $\varnothing \notin \mathcal{W}$;

- $N \in \mathcal{W}$;

- if $S \in \mathcal{W}$ and $S \subset T$, then $T \in \mathcal{W}$ (monotonicity proterty). 
$\mathcal{W}$ is called set of winning coalitions, and the couple $(N, \mathcal{W})$ represents the simple game.

The starting point in the above definition of simple game is that, each coalition of players is classified as either winning or losing. This is summarized by the characteristic function $V: 2^{N} \longrightarrow\{0,1\}$, where $V(S)=1$ if and only if the coalition $S$ is winning $(S \in \mathcal{W})$.

When players are seen as voters, $\mathcal{W}$ is called the voting rule and $V \equiv(N, \mathcal{W})$ the voting game. A weighted majority game is a voting game that can be represented by the symbol $\left[q ; w_{1}, w_{2}, \ldots, w_{n}\right]$ where $q>0$ is called the quota and $w_{i}>0$ is the weight associated with the $i^{t h}$ voter. In the standard case, voters make a yes or no decision on some political issue; and the issue is passed if the number of supporting votes overcomes the threshold q. The associated game's characteristic function $V$ satisfies the condition $V(S)=1$ if and only if $\sum_{i \in S} w_{i} \geqslant q$, for all coalitions $S \in 2^{N}$. For instance, the three most common voting games are the straight majority game represented by $[(n+1) / 2 ; 1,1, \ldots, 1]$, when $n$ is odd ; the pure bargaining game (or unanimity game) given by $\mathcal{W}=\{N\}$, but also represented by $[n ; 1,1, \ldots, 1]$, and the 3 -person market game $V_{3}(1)$ represented by $[3 ; 2,1,1]$.

\subsection{The theory of voting power}

A power index is a function $\boldsymbol{\psi}:=\left(\psi_{i}\right)_{i}$ which assigns the vector $\boldsymbol{\psi}(\boldsymbol{N}, \mathcal{W}) \in \mathbb{R}^{\boldsymbol{n}}$ to each simple game $(N, \mathcal{W})$, where each component $\psi_{i}(N, \mathcal{W})$ is the power of voter $i$ in $(N, \mathcal{W})$.

Definition 2 (decisive voter) A voter $i$ is said to be decisive or critical for a winning coalition $S$ if by leaving $S, i$ makes the remaining coalition $S \backslash\{i\}$ losing.

We will denote by $\mathcal{D}_{i}(\mathcal{W})$ the set of coalitions for which voter $i$ is decisive. A winning coalition $S$ is called minimal winning coalition if and only if every proper subset of $S$ is losing. We will also denote by $\mathcal{M}(\mathcal{W})$ (respectively $\mathcal{M}_{i}(\mathcal{W})$ ) the set of minimum winning coalitions (respectively the set of minimum winning coalitions containing voter $i$ ). Without any confusion, $\mathcal{D}_{i}(\mathcal{W}), \mathcal{M}(\mathcal{W})$ and $\mathcal{M}_{i}(\mathcal{W})$ will be rewritten as $\mathcal{D}_{i}, \mathcal{M}$ and $\mathcal{M}_{i}$. 
As mentioned in Brueckner (2001), in all well-known power indices, the power $\psi_{i}$ of voter $i$ is defined as the weighted sum of contributions $K_{i}\left(S_{i}\right)$ in a game $V$ that voter $i$ brings to each possible coalition $S_{i}$ in which s/he belongs to. The weighted factor for each coalition $S_{i}$ is denoted by $f\left(S_{i}\right)$. Formally,

$$
\psi_{i} \equiv \psi_{i}(V) \quad:=\sum_{S_{i}} f\left(S_{i}\right) K_{i}\left(S_{i}\right)
$$

where $K_{i}\left(S_{i}\right)$ depends on the specific definition of power in the coalition $S_{i}$ and in almost all standard power indices $K_{i}\left(S_{i}\right):=V\left(S_{i}\right)-V\left(S_{i} \backslash\{i\}\right)$, which is also called the marginal contribution of voter $i$ in the coalition $S_{i}$. On the other hand, the coefficient $f\left(S_{i}\right)$ depends on behavioral assumptions on the formation of coalitions.

In the next subsection, we are only concerned about three well-known power indices in the literature, namely, Shapley-Shubik index, Banzhaf-Coleman index and the Public Good index. First, let us begin by some recalls. We will define them with respect to the standard form provided by formula $2 \mathrm{~A}$.

\subsubsection{Standard approaches}

Symmetric Shapley-Shubik index (Shapley and Shubik, 1954) The ShapleyShubik index measures a voter's power by considering the relative expected frequency with which s/he turns a losing coalition into a winning one. Consider all possible orderings of $n$ voters (i.e permutations), which may be taken as all of the potential ways of building up towards the full coalition. Then, following any of these permutations, there is a unique voter who by joining the coalition, turns it from losing to winning: that voter is called the "pivot" of the considered permutation. Thus, the Shapley-Shubik index denoted $\phi:=\left(\phi_{i}\right)_{i}$ is, to any voter $i$, the frequency whereby $\mathrm{s} /$ he is the pivot over all possible permutations. Formally, for all $V \in \mathcal{G}_{N}$, we have from formula $2 \mathrm{~A}$,

$$
\phi_{i}=\psi_{i}, \text { where } K\left(S_{i}\right):=\left\{\begin{array}{ll}
1 & \text { if } S_{i} \in \mathcal{D}_{i} \\
0 & \text { otherwise }
\end{array}, \text { and } f\left(S_{i}\right):=\frac{\left(s_{i}-1\right) !\left(n-s_{i}\right) !}{n !}\right.
$$


Symmetric Banzhaf-Coleman index (Banzhaf III, 1964; and Coleman, 1971).

The non normalized Banzhaf-Coleman index denoted by $\overline{\boldsymbol{\beta}}:=\left(\bar{\beta}_{k}\right)_{k}$, is similar to the Shapley-Shubik index, but it considers the marginal contributions of a voter to all the coalitions s/he belongs to, without considering the order of the voters. More formally, for any voter $i \in N$ and any $V \in \mathcal{G}_{N}$, from formula 2A,

$$
\bar{\beta}_{i}=\psi_{i}, \text { where } K\left(S_{i}\right):=\left\{\begin{array}{ll}
1 & \text { if } S_{i} \in \mathcal{D}_{i} \\
0 & \text { otherwise }
\end{array} \text {, and } f\left(S_{i}\right):=\left(\frac{1}{2}\right)^{n-1} .\right.
$$

And its normalized version denoted by $\boldsymbol{\beta}:=\left(\beta_{i}\right)_{i}$, is defined for any voter $i$ by,

$$
\beta_{i}=\frac{\bar{\beta}_{i}}{\sum_{k \in N} \bar{\beta}_{i}}
$$

In this symmetric case, the normalized version is always efficient, but we will see next that, this efficiency property is no longer always guaranteed in the spatial model.

The Public Good index (Holler, 1982). The Public Good index or Holler index, denoted by $\gamma:=\left(\gamma_{i}\right)_{i}$ is defined as the normalization of the frequency for which a voter belongs to a minimal winning coalition. More formally, for any voter $i \in N$ and any $V \in \mathcal{G}_{N}$, from formula $2 \mathrm{~A}$,

$$
\gamma_{i}=\psi_{i}, \text { where } K\left(S_{i}\right):=\left\{\begin{array}{cc}
1 & \text { if } S_{i} \in \mathcal{M}_{i} \\
0 & \text { otherwise }
\end{array} \text {, and } f\left(S_{i}\right):=\frac{\left|\mathcal{M}_{i}\right|}{\sum_{k \in N}\left|\mathcal{M}_{k}\right|}\right.
$$

\subsubsection{Probabilistic interpretations}

As Shenoy (1982), we argue that one way of looking at how a voting system distributes power among its voters, is to suppose that each issue will induce a probability with which each voter will vote 'aye' for the issue. If a voter is strongly for the issue, then that probability will be close to 1 , if $\mathrm{s} /$ he is strongly against it, it will be close to 0 , and if $\mathrm{s} /$ he is indifferent to it, it will be close to $1 / 2$. Now, suppose that a decision making committee must decide to pass or reject a sequence of issues. Thus, related to each issue $\xi$, 
$p_{\xi}^{i}$ represents the probability that voter $i$ will vote 'aye' for $\xi$, and then, will be a member of the coalition supporting the issue $\xi$ with the probability $p_{\xi}^{i}$.

In the literature, there are several approaches about these individual probabilities. We usually consider independent assumption (i.e. all $p^{i}, i \in N$ are independent), homogeneity assumption (i.e. all $p^{i}, i \in N$ are equals) or partial homogeneity assumption (i.e. probabilities are identical within $m$ different groups, but independent across those groups). Sometimes, we consider uniformly distributions over these probabilities depending both on the assumption we have and what we are focusing on.

For instance, we will consider the homogeneity and independence assumptions from Brams et al. (p. 298); and after a slightly modification of the independence assumption highlighted in Leech (1990), we obtain the following three assumptions.

Independence assumption (A1). Every issue $\xi$ has a probability $p_{\xi}^{i}$ of appealing to the voter $i$. Each of the $p_{\xi}^{i}$ is chosen independently from the interval $[0,1]$ and such that the mean of the distribution is $1 / 2$. (Here, how one voter feels about an issue $\xi$ has nothing to do with how another one feels about the same issue. $)^{1}$

Homogeneity assumption (A2). Every issue $\xi$ to be decided on by the decision-making body has a certain probability $p_{\xi}$ of appealing to every single member of the committee. Furthermore, for various proposals, $p_{\xi}$ varies uniformly between 0 and 1 . (The homogeneity among the voters means that they all have the same probability $p_{\xi}$ of voting for the issue $\xi$, but $p_{\xi}$ varies from issue to issue.)

Partial homogeneity assumption (A3). This case is a mixture of the two assumptions above. Actually, for any issue $\xi$ the voter set $N$ is partitioned into $m$ groups such that individual probabilities on $\xi$ are identical within groups, but independent across groups. More formally, there exist a partition $\left(H_{k}\right)_{k=1, \ldots, m}$ of $N$, and a sequence $\left(q_{k}\right)_{k}$

\footnotetext{
${ }^{1}$ It should be noted that this assumption does not depend on the uniform distribution. It holds for any symmetric probability distribution from which the $p_{\xi}^{i}$ s are drawn independently.
} 
such that $q_{k} \sim U[0,1]$, and $p_{\xi}^{i}=q_{k}$ for all $i \in H_{k}, k=1, \ldots, m$.

Coalition formation with respect to issue $\xi$. The probability that a coalition $S_{i}$ containing voter $i$ forms with respect to any issue $\xi$, is denoted by $p_{\xi}\left(S_{i}\right)$. This represents the conditional probability of coalition formation $S_{i}$, given that voter $i$ supports the issue $\xi$ (i.e. conditional on $p_{\xi}^{i}=1$ ). Then, by considering all the available issues, the likelihood of the coalition formation of $S_{i}$ is given as $f\left(S_{i}\right)=\int_{\xi} p_{\xi}\left(S_{i}\right) d F_{\xi}$. Thus, by formula 2A

$$
\psi_{i}=\sum_{S_{i}} f\left(S_{i}\right) K_{i}\left(S_{i}\right)=\int_{\xi} \Psi_{i}(\xi) d F_{\xi}, \text { where } \Psi_{i}(\xi):=\sum_{S_{i}} p_{\xi}\left(S_{i}\right) K_{i}\left(S_{i}\right)
$$

Now, taking into account our three assumptions (A1), (A2), (A3), it yields the followings.

- Under the independence assumption (A1) on issue $\xi, p_{\xi}\left(S_{i}\right)$ is given by,

$$
p_{\xi}\left(S_{i}\right):=\prod_{j \in S_{i} \backslash\{i\}} p_{\xi}^{j} \prod_{k \in N \backslash S_{i}}\left(1-p_{\xi}^{k}\right) .
$$

Since the mean of the probability distribution of each $p_{\xi}^{i}$ on $[0,1]$ is $1 / 2$, we obtain:

$$
f\left(S_{i}\right)=\int_{\xi} p_{\xi}\left(S_{i}\right) d F_{\xi}=\prod_{j \in S_{i} \backslash\{i\}} \int_{0}^{1} p^{j} d p^{j} \prod_{k \in N \backslash S_{i}} \int_{0}^{1}\left(1-p^{k}\right) d p^{k}=\left(\frac{1}{2}\right)^{n-1} .
$$

In particular, when for all $k \in N \backslash\{i\}, p_{\xi}^{k}=1 / 2$, we obviously obtain,

$$
f\left(S_{i}\right)=\int_{\xi} p_{\xi}\left(S_{i}\right) d F_{\xi}=\left(\frac{1}{2}\right)^{n-1}
$$

- Under the homogeneity assumption (A2), it follows that,

$$
p_{\xi}\left(S_{i}\right) \quad:=\prod_{j \in S_{i} \backslash\{i\}} p_{\xi} \prod_{k \in N \backslash S_{i}}\left(1-p_{\xi}\right)=p_{\xi}^{s_{i}-1}\left(1-p_{\xi}\right)^{n-s_{i}} .
$$

Thus, if the probability distribution on issues implies that $p_{\xi} \sim U[0,1]$, then

$$
\begin{aligned}
f\left(S_{i}\right)=\int_{\xi} p_{\xi}\left(S_{i}\right) d F_{\xi}=\int_{\xi} p_{\xi}^{s_{i}-1}\left(1-p_{\xi}\right)^{n-s_{i}} d F_{\xi} & =\int_{0}^{1} p^{s_{i}-1}(1-p)^{n-s_{i}} d p \\
& \overline{\overline{(*)}} \frac{\left(s_{i}-1\right) !\left(n-s_{i}\right) !}{n !}
\end{aligned}
$$


Where $(*)$ is because of Beta integral's properties, and $s_{i}:=\left|S_{i}\right|$.

- Under the partial homogeneity assumption (A3), we will obtain,

$$
p_{\xi}\left(S_{i}\right) \quad:=\left[\prod_{\substack{H_{l} \ni i \\ j \in H_{l} \cap S_{i} \backslash\{i\}}} q_{\xi}^{l} \prod_{\substack{k \in H_{l} \\ k \in N \backslash S_{i}}}\left(1-q_{\xi}^{l}\right)\right] \times \prod_{r \neq l}\left[\prod_{j \in H_{r} \cap S_{i} \backslash\{i\}} q_{\xi}^{r} \prod_{\substack{k \in H_{r} \\ k \in N \backslash S_{i}}}\left(1-q_{\xi}^{r}\right)\right] .
$$

Interpretation. In terms of probabilistic interpretation, the coefficient $f\left(S_{i}\right)$ can be interpreted as the probability that the coalition $S_{i}$ will form taking under consideration all the issues. Once such issues are known, then the power index obtained is subsequently said to be asymmetric; in particular when these issues come from spatial models as we will see in the next section, those asymmetric power indices are thereby called spatial power indices.

By contrast, in absence of any information about these issues, we will usually consider the two first aforementioned assumptions (A1) and (A2). Consequently, obtain the four propositions stated below ${ }^{2}$, which also use the two following formulae,

$$
K\left(S_{i}\right) \quad:=\left\{\begin{array}{ll}
1 & \text { if } S_{i} \in \mathcal{D}_{i} \\
0 & \text { otherwise }
\end{array},\right.
$$

and

$$
K\left(S_{i}\right) \quad:=\left\{\begin{array}{ll}
1 & \text { if } S_{i} \in \mathcal{M}_{i} \\
0 & \text { otherwise }
\end{array} .\right.
$$

Here are our propositions.

Proposition 1 The independence assumption (A1), along with the definition (2B) and the relation $(\mathbf{2 F})$, yield the symmetric non-normalized Banzhaf power index.

Proposition 2 The homogeneity assumption (A2), along with the definition (2B) and the relation (2F), yield the symmetric Shapley-Shubik power index.

Proposition 3 The independence assumption (A1), along with the definition (2B) and the relation (2G), yield the symmetric Public Good power index (after normalization).

\footnotetext{
${ }^{2}$ Actually, by considering the partial homogeneity assumption (A3), one might also add two more propositions (one related to Shapley-Shubik, and the other to the Public Good index); but both are irrelevant for our model.
} 
Proposition 4 The homogeneity assumption (A2), along with the definition (2B) and the relation (2G), yield what is called the symmetric Public Good power index for homogeneous voting behavior (PGH for short) (after normalization).

\section{Spatial Games}

These kinds of games are simply defined as voting games associated with a subdomain $\mathbb{D}$ of the $m$ - dimensional Euclidean space $\mathbb{R}^{m}$. Usually, spatial voting games represent in the best way the framework for political games, whereby the voters are assumed to have their most preferred points in the space $\mathbb{D}$. These points, representing those voters, are called their ideal points ${ }^{3}$, and the space $\mathbb{D}$ is henceforth called ideological space of the spatial game. Actually, in $\mathbb{R}^{m}$, each dimension represents political or ideological parameters, such as, left/right (economic), left/right (international), economic growth/environment, etc. (e.g when $m=1$ the linear structure of $\mathbb{R}$ will help us to capture intuitive ideas like moderation and extremism, then we can think of $\mathbb{R}^{1}$ as the left to right spectrum of political ideology). Moreover, the conventional assumption made is that, voters have euclidean preferences - the more a point is close to my ideal point, the more I prefer that point; in another words, voters prefer points that are close to their ideal points.

From now on, we suppose that each voter $i \in N$ is represented by $Q^{i} \in \mathbb{D}$, with $\mathbb{D} \subseteq \mathbb{R}^{m}$.

Definition $3 A$ spatial game $\mathcal{V}$ defined on an ideological space $\mathbb{D} \subseteq \mathbb{R}^{m}$, is a triplet $\left(N, \mathcal{W}, Q_{N}\right)$, satisfying the following two items.

1. $(N, \mathcal{W})$ is a simple voting game, with a characteristic function $V: 2^{N} \longrightarrow\{0,1\}$ such that $V(S)=1$ if and only if $S \in \mathcal{W}$;

2. $\boldsymbol{Q}_{N}:=\left(Q^{i}\right)_{i \in N}$ is the vector of ideal points, each of them belonging to $\mathbb{D}$.

We denote the spatial game in the ideological space $\mathbb{D} \subset \mathbb{R}^{m}$ by $\mathcal{V}(\mathbb{D}):=\left(N, \mathcal{W}, Q_{N}\right)$.

\footnotetext{
${ }^{3}$ They represent ideological description or political profile of voters.
} 


\subsection{Ideology and issue}

Here, we will recall the model that is defined by Shenoy (1982) by keeping in mind that, the purpose of this subsection is to provide the probabilities $p_{\xi}^{i}$ of voters $i \in N$, given their ideological positions $Q^{i}$ along with the political issues $\xi$. First, consider the ideological space $\mathbb{D}$ as being the half-ball $\mathbb{B}_{1 / 2}^{m}$ recalled below.

$$
\mathbb{B}_{1 / 2}^{m}:=\left\{x \in \mathbb{R}^{m}:\left(x_{1}^{2}+x_{2}^{2}+\cdots+x_{m}^{2}\right)^{1 / 2} \leqslant \frac{1}{2}\right\} .
$$

Notice that, each ideal point (i.e political profile) must belong to $\mathbb{B}_{1 / 2}^{m}$. Moreover, the collection of those points is called a constellation, denoted by $C(N)$ and defined as follows.4

$$
C(N):=\quad\left\{x \in \mathbb{B}_{1 / 2}^{m} / \exists i \in N: x=Q^{i}\right\}
$$

The second stage, is the way political issues are represented. In the Shenoy]s computation scheme, it is assumed that, the assembly of voters will be presented with a series of issues that arise out of some random process. Thus, in this spatial model, each issue $\xi$ will consist of some combination of the parameters of the ideological space, and will generate for any voter $i$ the probability $p_{\xi}^{i}$ with which s/he will vote 'aye' on $\xi$, and $p_{\xi}^{i}$ is given by:

$$
p_{\xi}^{i} \quad:=\frac{1}{2}+\xi_{1} Q_{1}^{i}+\xi_{2} Q_{2}^{i}+\cdots+\xi_{m} Q_{m}^{i}
$$

Here, the issue $\xi:=\left(\xi_{1}, \xi_{2}, \ldots, \xi_{m}\right)$ will always be considered as a unit vector. In other words, the set of political issues is the unit hypersphere $\mathbb{S}_{1}^{m}$ defined as,

$$
\mathbb{S}_{1}^{m}:=\quad\left\{x \in \mathbb{R}^{m}:\left(x_{1}^{2}+x_{2}^{2}+\cdots+x_{m}^{2}\right)^{1 / 2}=1\right\}
$$

From $3 \mathrm{~B}$, it follows that $p_{\xi}^{i} \in[0,1]$, as satisfied by probabilities: so $p_{\xi}^{i}$ it is well-defined.

As stated in Shapley (1977), both the political profiles and the political issues, represented as points in the $m$-dimensional space, should be regarded differently as belonging to dual spaces, not to the same. For a visual illustration, we refer the reader to Figure 1 .

\footnotetext{
${ }^{4}$ So unlike the Shapley s approach or even approaches in Owen (1971), Rapoport and Golan (1985), Alonso-Meijide et al. (2011), any two voters here, are allow to choose the same ideal points. Which by the way, makes sense in real situations.
} 


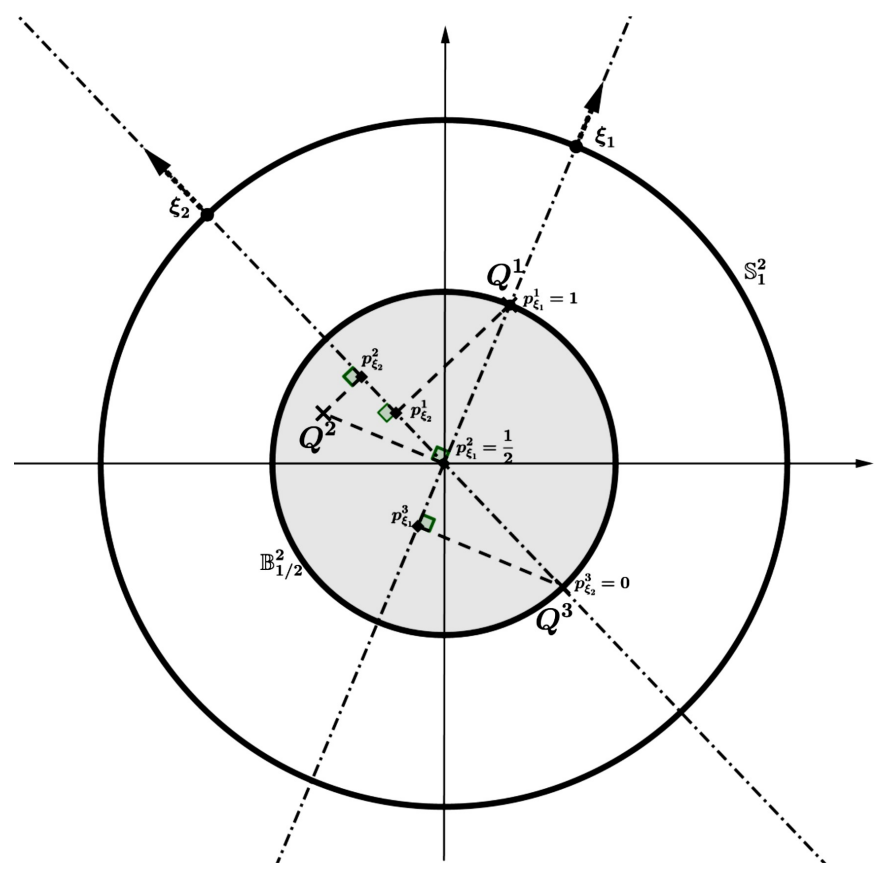

Figure 1: ideological space $\mathbb{B}_{1 / 2}^{m}$, and issues space $\mathbb{B}_{1}^{m}$, when $m=2$.

\subsection{Spatial power indices}

In this subsection we will define some spatial power indices on any given voting rule, and these definitions will take into account both the constellation $C(N)$ followed by political issues. Since we do not have any additional information about political issues other than the issue's space itself, we will mostly assume that each issue is randomly selected according to the uniform probability distribution $\left.\right|^{5}$ on the unit hypersphere $\mathbb{S}_{1}^{m}$. On the other hand, without any further information about political profiles on voters, we will obviously suppose that they are all independent. Obviously, as the voters are supposed to have independent ideal points $\left\{Q^{i}, i \in N\right\}$, the probabilities $\left\{p_{\xi}^{i}\right\}_{i \in N}$ on any issue $\xi$ are also independent. That is why, by taking into account this independence assumption, the spatial approach in this paper is more appropriate for both the Banzhaf index and the Public good index, as the above propositions show.

\footnotetext{
${ }^{5}$ If in some particular context, this assumption is not satisfied, then we must use an appropriate distribution function on $\mathbb{S}_{1}^{m}$. (especially when we have precise information about political issues)
} 
Shenoy is the pioneer of the spatial approach of this paper, and he proposed the Banzhaf spatial index ${ }^{6}$ which is based on the Question of effect on outcome developed in Straffin (1977), p.112; that is why, the spatial model requires that in absence of information that, both the political profiles must be independent, while the political issues $\xi \in \mathbb{S}_{1}^{m}$ must be drawn from the uniform probability distribution. In order to integrated in the same spatial model the Public Good indices, we need to consider a modified version of that "Question of effect on outcome". Actually, that previous question combines at the same time the probability of formation of coalitions and the concept of power, whereas, based on Brueckner (2001), our work treats these two effects separately, as made in subsection 2.2.2 so that restricted to the Shenoy's assumption, we exactly obtain the same Banzhaf spatial index. Below are provided our findings.

\subsubsection{Banzhaf spatial power index}

Denoted by $\overline{\boldsymbol{\psi}}^{\boldsymbol{B}}:=\left(\bar{\psi}_{i}^{B}\right)_{i}$, the Banzhaf spatial index is defined for each voter $i$, with respect to the previous spatial model as follows,

$$
\bar{\psi}_{i}^{B}:=\int_{\xi} \Psi_{i}^{B}(\xi) d F_{\xi}, \quad \text { with } \Psi_{i}^{B}(\xi):=\sum_{S_{i}} p_{\xi}\left(S_{i}\right) K_{i}\left(S_{i}\right),
$$

where the issues $\xi$ are drawn from the uniform distribution on $\mathbb{S}_{1}^{m}$, and $F_{\xi}$ being the uniform cumulative probability distribution function on $\mathbb{S}_{1}^{m}$. Furthermore, the factor $p_{\xi}\left(S_{i}\right)$ is given by formula $(2 \mathrm{C})$ while $K_{i}\left(S_{i}\right)$ is given by formula $\left.2 \mathrm{~F}\right)$, both leading to the next equality.

$$
\Psi_{i}^{B}(\xi)=\sum_{S \in \mathcal{D}_{i}} \prod_{j \in S \backslash\{i\}} p_{\xi}^{j} \times \prod_{k \in N \backslash S}\left(1-p_{\xi}^{k}\right), \quad \text { for all } \xi \in \mathbb{S}^{m} .
$$

Notice that, when all the ideal points are located at $O$ (the center of the half-ball $\mathbb{B}_{1 / 2}^{m}$ ), we obtain $p_{\xi}^{i}=1 / 2$, for all voter $i$ and issue $\xi$. Thus, the Banzhaf spatial index will coincide with the symmetrical Banzhaf index, regardless of the probability distribution 7

\footnotetext{
${ }^{6}$ After many discussion with some researchers including Shapley, as claimed the author.

${ }^{7}$ This coherence does not appear in Owen-Shapley index (Shapley, 1977), where it is "quite hard" to get back to the classic Shapley-Shubik index, especially in higher dimensions, since it requires $n=m$ for coinciding with its classic form.
} 
The normalized Banzhaf spatial index, denoted by $\boldsymbol{\psi}^{B}:=\left(\psi^{B}{ }_{i}\right)_{i}$, is defined for each voter $i$ by:

$$
\psi_{i}^{B}:= \begin{cases}\frac{\bar{\psi}_{i}^{B}}{\sum_{k \in N} \bar{\psi}_{k}^{B}} & \text { if } \sum_{k \in N} \bar{\psi}_{k}^{B} \neq 0 \\ 0 & \text { otherwise }\end{cases}
$$

We obviously check that $\boldsymbol{\psi}^{B}$ is efficient 8 if and only if $\sum_{k \in N} \bar{\psi}_{k}^{B} \neq 0$. In practice, due to the appealing property of the efficiency, we will mostly use $\boldsymbol{\psi}^{\boldsymbol{B}}$ rather than $\overline{\boldsymbol{\psi}}^{\boldsymbol{B}}$.

\subsubsection{The Public Good spatial power index}

For this new index, called the non normalized Public Good spatial index and denoted by $\overline{\boldsymbol{\psi}}^{P G}:=\left(\bar{\psi}_{k}^{P G}\right)_{k}$, the assumptions made about political profiles and political issues remain the same as those of the Banzhaf spatial index. The only difference behind this new index, comes from the coalition formation as stated previously about the standard Public Good index and minimal winning coalitions. We will now formally define our new spatial index for any voter $i$ by,

$$
\bar{\psi}_{i}^{P G}:=\int_{\xi} \Psi_{i}^{P G}(\xi) d F_{\xi}, \quad \text { with } \Psi_{i}^{P G}(\xi):=\sum_{S_{i}} p_{\xi}\left(S_{i}\right) K_{i}\left(S_{i}\right),
$$

where the issues $\xi$ are drawn from the uniform distribution on $\mathbb{S}_{1}^{m}$, and $F_{\xi}$ being the uniform cumulative probability distribution function on $\mathbb{S}_{1}^{m}$. Furthermore, the factor $p_{\xi}\left(S_{i}\right)$ is given by formula $(2 \mathrm{C})$ while $K_{i}\left(S_{i}\right)$ is given by formula $(2 \mathrm{G})$, both leading to the next equality:

$$
\Psi_{i}^{P G}(\xi)=\sum_{S \in \mathcal{M}_{i}} \prod_{j \in S \backslash\{i\}} p_{\xi}^{j} \times \prod_{k \in N \backslash S}\left(1-p_{\xi}^{k}\right), \quad \text { for all } \xi \in \mathbb{S}^{m} .
$$

Furthermore, one can realize that, our new spatial index always coincides with the Banzhaf spatial index when $\mathcal{D}_{i}=\mathcal{M}_{i}$ for all voter $i$. That would evidently happen whenever all the voters are symmetric in the voting game.

Now, we define the Public Good spatial index denoted by $\boldsymbol{\psi}^{\boldsymbol{P G}}$ as being the normalized version of $\overline{\boldsymbol{\psi}}^{\boldsymbol{P G}}$. To wit, for all voter $i$

\footnotetext{
${ }^{8} \mathrm{~A}$ power index is efficient when by summing up all its components in any simple game, we always obtain 1 .
} 


$$
\psi_{i}^{P G}:=\left\{\begin{array}{ll}
\frac{\bar{\psi}_{i}^{P G}}{\sum_{k \in N} \bar{\psi}_{k}^{P G}} & \text { if } \sum_{k \in N} \bar{\psi}_{k}^{P G} \neq 0 \\
0 & \text { otherwise }
\end{array} .\right.
$$

Here again, $\boldsymbol{\psi}^{P G}$ is efficient if and only if $\sum_{k \in N} \bar{\psi}_{k}^{P G} \neq 0$. This implies that, the efficiency is not always satisfied as it does in the symmetric Public Good index 9 . Also, it could be worth mentioning that, once all the ideal points are located at point $O$ (i.e $p_{\xi}^{i}=1 / 2, i \in$ $\left.N ; \xi \in \mathbb{S}_{1}^{m}\right)$, the new Public Good spatial index will always coincide with its symmetric form (this result is independent of any probability distribution on $\mathbb{S}_{1}^{m}$ ).

Remark: one might realize that on symmetric games, the Public Good spatial power index always coincides with the normalized Banzhaf spatial power index. Examples of such cases, using straight majority games and pure bargaining games, are provided in Section 4

\subsubsection{The Shapley-Shubik index and the Public Good index with homogeneity}

So far, one might realize that we were focused on power indices linked to independence assumptions, and might ask what about those related to homogeneity assumptions? Before answering the question, let us first clarify that, by the Public Good index with homogeneity, we mean the one related to homogeneity assumption as mentioned in Proposition 4. and abbreviated by $P G H$. Now, the question that can help us is: "under what conditions in our spatial approach, do we establish the Shapley-Shubik index and the PGH index?" More generally, related to the probabilities $p_{\xi}^{i}$, under what conditions do we obtain the homogeneity assumption (A2)? When $m=2$, an answer is given by the following theorem 10

Theorem 5 For $m=2$, if all the voters are located at the point $(1, \gamma), 0 \leqslant \gamma<2 \pi$ (in polar coordinates); and the probability distribution of issues $\xi:=(1, \theta)$ is given by the

\footnotetext{
${ }^{9}$ For a counterexample, consider the straight majority rule in which, for any voter $i$ and issue $\xi$, $p_{\xi}^{i} \in\{0,1\}$.

${ }^{10}$ This theorem that we do not proof here, is a slightly modification of the one in Shenoy, 1982, p.307
} 
density function

$$
f_{\xi}(\theta) \quad:=\frac{1}{4}|\sin (\theta-\gamma)|, \quad \forall \theta \in[0,2 \pi[.
$$

Then, for all $i \in N, p_{\xi}^{i}=p_{\theta} \sim U[0,1]$, with $p_{\theta}:=\frac{1}{2} \cos (\theta-\gamma)$.

The result of this theorem is straightforward interpreted as being the homogeneity assumption (A2). For higher dimensions, such a theorem is still possible. The voters just have to be located altogether at any point on the boundary of $\mathbb{B}_{1 / 2}^{m}$. Additionally, the probability distribution of issue directions $\xi \in \mathbb{S}_{1}^{m}$ must be such that, the induced probability distribution of $p_{\xi}$ belongs to $U[0,1]$. Then, we will have the homogeneity assumption (A2) accordingly ${ }^{11}$.

\section{Illustration by a few examples}

We notice that in real case studies the dimension $m$ of data (political profiles and political issues) is almost always large $(m>>3)$. Thus, in such cases, we usually use some dimensionality reduction techniques $[12$, which allow us to reduce the original dimension of the data space so that we can obtain in smaller dimensions (usually dimensions 1,2 or 3), a pretty good representation of our data. Thus, we need to know how to compute our spatial indices in such lower dimensions. Accordingly, in the following we are interested in dimensions 1, 2 and 3, to illustrate the computation of spatial indices through our method. Some examples are also given afterwards.

\footnotetext{
${ }^{11}$ If we were concerned about indices based on assumption (A3) (like the one use in Straffin $(1977)$ for the study of the Canadian constitutional amendment scheme ; and the ones used in Brueckner (2001)), we would have in an appropriate form, a similar result as this theorem for 3-dimensional space and higher. But this is beyond the scope of this paper. Furthermore, as already mentioned, this spatial model is by construction, best fitted under assumption (A1).

${ }^{12}$ E.g. the most well-known is the so-called factor analysis methods, the interested reader can find out more in Harman (1976)
} 


\subsection{Illustrations}

In the next three claims, any issue is denoted by $\xi \in \mathbb{S}_{1}^{m}$, and the ideal point of any voter $i \in N$ is denoted by $Q^{i}:=\left(Q_{1}^{i}, Q_{2}^{i}, \ldots, Q_{m}^{i}\right) \in \mathbb{B}_{1 / 2}^{m}$. Furthermore, whether the spatial power index $\boldsymbol{\beta}$ is Banzhaf or the Public Good (non normalized), for any voter $i, \beta_{i}$ is given by the following expression,

$$
\beta_{i}=\int_{\xi} \Psi_{i}(\xi) d F_{\xi} \equiv \int_{\xi} \Psi_{i}(\xi) f_{\xi} d \xi
$$

where $f_{\xi}$ is the density function associated to the uniform distribution of issues on $\mathbb{S}_{1}^{m}$.

From now on, what matters in practice is the way each probability is really obtained.

Claim 1: In 1-dimension, we consider euclidean coordinates.

Here, for any voter $i, i$ 's probability of agreement on any issue $\xi \in\{-1,1\}$ is given by:

$$
p_{\xi}^{i}=\frac{1}{2}+Q^{i} \xi, \quad \text { where } Q^{i} \in \mathbb{B}_{1 / 2}^{1}=\left[-\frac{1}{2}, \frac{1}{2}\right]
$$

Thus,

$$
\beta_{i}=\frac{1}{2}\left[\Psi_{i}(-1)+\Psi_{i}(1)\right] .
$$

Claim 2: In 2-dimension, we consider polar coordinates.

Here, $i$ 's probability of agreement on issues $\xi:=(1, \theta),(0 \leqslant \theta<2 \pi)$ is given by:

$$
p_{\xi}^{i}=\frac{1}{2}+r_{i} \cos \left(\theta-\theta_{i}\right), \quad \text { where } Q^{i} \in \mathbb{B}_{1 / 2}^{2} ; \text { with } Q^{i}:=\left(r_{i}, \theta_{i}\right)
$$

Then,

$$
\beta_{i}=\frac{1}{2 \pi} \int_{0}^{2 \pi} \Psi_{i}(\theta) d \theta
$$

Claim 3: In 3-dimension, we consider spherical coordinates.

Here, with $\xi:=(1, \theta, \phi)$ and $Q^{i}:=\left(r_{i}, \theta_{i}, \phi_{i}\right) \in \mathbb{B}_{1 / 2}^{3} ; \quad 0 \leqslant \theta<2 \pi, 0 \leqslant \phi \leqslant \pi$, we get,

$$
p_{\xi}^{i}=\frac{1}{2}+r_{i} \cos \left(\theta-\theta_{i}\right) \cos \left(\phi-\phi_{i}\right), \quad \text { where } f_{\xi} \equiv f(\theta, \phi):=\frac{1}{4 \pi} \sin \phi .
$$

Therefore,

$$
\beta_{i}=\frac{1}{4 \pi} \int_{0}^{2 \pi}\left(\int_{0}^{\pi} \Psi_{i}(\theta, \phi) \sin \phi d \phi\right) d \theta
$$




\subsection{Examples of symmetric 3-voters voting games}

This subsection provides two distinguished examples on classic games. Both of them are given in dimensions one and two, and also consider the three voters as being 1,2 and 3, along with their respective ideal points $Q^{1}, Q^{2}$ and $Q^{3}$.

\subsubsection{Straight majority voting rule}

Here, the voting rule is given by $\mathcal{M}:=\{12,13,23\}$. As we see, voters are all symmetric, which implies that the normalized Banzhaf spatial index coincides with the Public Good spatial index. In order to compute the power of voters, notice that, related to any $\xi \in \mathbb{S}_{1}^{m}$, we have,

$$
\Psi_{i}(\xi):=p_{\xi}^{j} \times\left(1-p_{\xi}^{k}\right)+\left(1-p_{\xi}^{j}\right) \times p_{\xi}^{k}, \quad \text { for } i, j, k=1,2,3 \quad ; \quad i \neq j ; j \neq k ; k \neq i .
$$

In dimension 1 (i.e. $m=1$ ): by $4 \mathrm{~A}$ and Claim 1 , we have for each voter $i$,

$$
\begin{aligned}
\Psi_{i}(\xi) & =\left(\frac{1}{2}+\xi Q^{j}\right)\left(\frac{1}{2}-\xi Q^{k}\right)+\left(\frac{1}{2}+\xi Q^{k}\right)\left(\frac{1}{2}-\xi Q^{j}\right) \\
& \overline{\overline{(*)}} \quad \frac{1}{2}-2 Q^{j} Q^{k}
\end{aligned}
$$

Where $(*)$ takes into account that $\xi^{2}=1$. Thus, using Claim 1 again, we obtain,

$$
\beta:=\left(\beta_{1}, \beta_{2}, \beta_{3}\right)=\left(\frac{1}{2}-2 Q^{2} Q^{3}, \frac{1}{2}-2 Q^{1} Q^{3}, \frac{1}{2}-2 Q^{1} Q^{2}\right) .
$$

In dimension 2 (i.e. $m=2$ ): by $4 \mathrm{~A}$ and Claim 2 , we have for each voter $i$,

$$
\begin{aligned}
\Psi_{i}(\xi)= & \left(\frac{1}{2}+r_{j} \cos \left(\theta-\theta_{j}\right)\right)\left(\frac{1}{2}-r_{k} \cos \left(\theta-\theta_{k}\right)\right) \\
& +\left(\frac{1}{2}+r_{k} \cos \left(\theta-\theta_{k}\right)\right)\left(\frac{1}{2}-r_{j} \cos \left(\theta-\theta_{j}\right)\right) .
\end{aligned}
$$

Here from Claim 2, we can write,

$$
\beta_{i}=\int_{\xi} \Psi_{i}(\xi) f_{\xi}=\frac{1}{2 \pi} \int_{0}^{2 \pi}\left(\Delta_{j k}(\theta)+\Delta_{k j}(\theta)\right) d \theta
$$


Where $\Delta_{j k}(\theta):=\left(\frac{1}{2}+r_{j} \cos \left(\theta-\theta_{j}\right)\right)\left(\frac{1}{2}-r_{k} \cos \left(\theta-\theta_{k}\right)\right)$, and we also have

$$
\Delta_{j k}(\theta)=\frac{1}{4}+\frac{1}{2}\left[r_{j} \cos \left(\theta-\theta_{j}\right)-r_{k} \cos \left(\theta-\theta_{k}\right)\right]-r_{j} r_{k} \cos \left(\theta-\theta_{j}\right) \cos \left(\theta-\theta_{k}\right) .
$$

Since,

$$
\int_{0}^{2 \pi} \cos \left(\theta-\theta_{j}\right) \cos \left(\theta-\theta_{k}\right) d \theta \overline{\overline{(*)}} \frac{1}{2} \int_{0}^{2 \pi}\left(\cos \left[2 \theta-\left(\theta_{j}+\theta_{k}\right)\right]+\cos \left(\theta_{j}-\theta_{k}\right)\right) d \theta
$$

and

$$
\int_{0}^{2 \pi} \cos \left(\theta-\theta_{j}\right) d \theta=0, \text { and } \int_{0}^{2 \pi} \cos \left[2 \theta-\left(\theta_{j}+\theta_{k}\right)\right] d \theta=0 \quad j, k=1,2,3 .
$$

Then, combining (4C), 4D) and 4E) yields to,

$\frac{1}{2 \pi} \int_{0}^{2 \pi} \Delta_{j k}(\theta) d \theta=\frac{1}{4}-\frac{1}{2} r_{j} r_{k} \cos \left(\theta_{j}-\theta_{k}\right) \overline{\overline{(\dagger)}} \frac{1}{4}-\frac{1}{2}\left(r_{j}, \theta_{j}\right)\left(r_{k}, \theta_{k}\right)=\frac{1}{4}-\frac{1}{2}\left(Q_{1}^{j} Q_{1}^{k}+Q_{2}^{j} Q_{2}^{k}\right)$

Notice that $(*)$ and $(\dagger)$ are respectively obtained from the following classic formulae:

$\cos (a) \times \cos (b)=\frac{1}{2}(\cos (a+b)+\cos (a-b)) \quad$ and $\quad(r, \phi) \cdot\left(r^{\prime}, \phi^{\prime}\right)=r r^{\prime} \cos \left(\phi-\phi^{\prime}\right)$.

Now, it remains to consider $4 \mathrm{~B}$ ) and $4 \mathrm{~F}$ to obtain the following result,

$$
\beta=\left(\frac{1}{2}-\left(Q_{1}^{2} Q_{1}^{3}+Q_{2}^{2} Q_{2}^{3}\right), \frac{1}{2}-\left(Q_{1}^{1} Q_{1}^{3}+Q_{2}^{1} Q_{2}^{3}\right), \frac{1}{2}-\left(Q_{1}^{1} Q_{1}^{2}+Q_{2}^{1} Q_{2}^{2}\right)\right)
$$

\subsubsection{The pure bargaining game (unanimity game)}

Because the corresponding rule is defined by $\mathcal{W}:=\{N\}$, all the voters are symmetric one another, which as previously implies that the normalized Banzhaf spatial index coincides with the spatial Public Good index. We will first consider three voters, and thereafter, we will generalize the result to an arbitrary number $n$ of voters, in both dimensions $(m=1$ and $m=2$ ). Voters are denoted by natural numbers and for all $i \in N, Q^{i}$ is the $i$ 's ideal point. Now, related to any political issue $\xi \in \mathbb{S}_{1}^{m}$, in the case on three voters 1,2 and 3 , we have,

$$
\Psi_{i}(\xi) \quad:=p_{\xi}^{j} \times p_{\xi}^{k}, \quad \text { for } i, j, k=1,2,3 \quad ; \quad i \neq j \neq k \neq i
$$


In dimension 1 (i.e. $m=1$ ): from $4 \mathrm{G}$ and Claim 1, we have for voter $i$,

$$
\Psi_{i}(\xi) \quad \overline{\overline{(*)}} \quad \frac{1}{4}+Q^{j} Q^{k}+\xi\left(Q^{j}+Q^{k}\right), \quad \text { with } \xi \in\{-1,1\} .
$$

Where $(*)$ means by the same method used in straight majority rule in dimension 1 , and again, by Claim 1 we obtain the following,

$$
\boldsymbol{\beta}:=\left(\beta_{1}, \beta_{2}, \beta_{3}\right)=\left(\frac{1}{4}+Q^{2} Q^{3}, \frac{1}{4}+Q^{1} Q^{3}, \frac{1}{4}+Q^{1} Q^{2}\right) .
$$

Generally, when the total number of voters is $n \geqslant 3$, we obtain by induction on $n$,

$$
\beta_{i}=\left(\frac{1}{2}\right)^{n-1}+\delta_{\{n \geqslant 4\}} \sum_{k=1}^{\left[\frac{n}{2}\right]-1}\left(\frac{1}{2}\right)^{n-1-2 k}\left[\sum_{\substack{S \subseteq N \backslash i \\|S|=2 k}} \prod_{j \in S} Q^{j}\right]+\left(\frac{1+(-1)^{n-1}}{2}\right) \times \prod_{j \in N \backslash i} Q^{j}
$$

In dimension 2 (i.e. $m=2$ ): from $4 \mathrm{G}$ and Claim 2, we have for voter $i$,

$$
\Psi_{i}(\xi)=\left(\frac{1}{2}+r_{j} \cos \left(\theta-\theta_{j}\right)\right)\left(\frac{1}{2}+r_{k} \cos \left(\theta-\theta_{k}\right)\right) .
$$

However, from Claim 2,

$$
\beta_{i}=\int_{\xi} \Psi_{i}(\xi) f_{\xi}=\frac{1}{2 \pi} \int_{0}^{2 \pi} \Psi_{i}(\theta) d \theta
$$

Which by a similar method used in the straight majority's case above, gives

$$
\begin{aligned}
\boldsymbol{\beta} & =\left(\beta_{1}, \beta_{2}, \beta_{3}\right) \\
& =\left(\frac{1}{4}+\frac{1}{2}\left(Q_{1}^{2} Q_{1}^{3}+Q_{2}^{2} Q_{2}^{3}\right), \frac{1}{4}+\frac{1}{2}\left(Q_{1}^{1} Q_{1}^{3}+Q_{2}^{1} Q_{2}^{3}\right), \frac{1}{4}+\frac{1}{2}\left(Q_{1}^{1} Q_{1}^{2}+Q_{2}^{1} Q_{2}^{2}\right)\right) .
\end{aligned}
$$

Similarly, when the total number of voters is $n \geqslant 3$, we obtain by induction on $n$,

$$
\forall k \in N, \quad \beta_{k}=\left(\frac{1}{2}\right)^{n-1}+\left(\frac{1}{2}\right)^{n-2} \sum_{1 \leqslant i<j \leqslant n ; i \neq k, j \neq k}\left(Q_{1}^{i} Q_{1}^{j}+Q_{2}^{i} Q_{2}^{j}\right) .
$$

\subsubsection{Some interpretations in dimension 1}

As we have already said, in symmetric games, our two indices are the same. Let us have a look at the two previous examples in dimension 1 , by considering various constellations as shown the table 1 . 
First, the way we must read table 1 (and table 2) can be intuitively think of as follows. About the position of constellations in both tables 1 and 2 , the extreme left position $(x=-1 / 2)$ is seen as the extreme left position in a political spectrum, similarly for the extreme right position $(x=+1 / 2)$, and also for the (absolute) centrist position $(x=0)$. One might even interpret such representations in the scope of relative positions (we would therefore have various values of power since they all depend on relative positions). Another mention is that, non separated points like $Q^{1} Q^{2}$ means they are in the same position in the space.

\begin{tabular}{|c|c|c|c|c|c|}
\hline \multirow[t]{2}{*}{$\#$} & \multirow{2}{*}{$\begin{array}{c}\text { Various } \\
\text { Constellations }\end{array}$} & \multicolumn{2}{|c|}{$\begin{array}{l}\text { Spatial power index } \beta \\
\text { (Straight Majority) }\end{array}$} & \multicolumn{2}{|c|}{$\begin{array}{l}\text { Spatial power index } \beta \\
\text { (Unanimity Game) }\end{array}$} \\
\hline & & $\left(\bar{\psi}_{1}, \bar{\psi}_{2}, \bar{\psi}_{3}\right)$ & $\left(\psi_{1}, \psi_{2}, \psi_{3}\right)$ & $\left(\bar{\psi}_{1}, \bar{\psi}_{2}, \bar{\psi}_{3}\right)$ & $\left(\psi_{1}, \psi_{2}, \psi_{3}\right)$ \\
\hline 1 & $Q^{1} Q^{2} Q^{3}$ & $\left(\frac{1}{2}, \quad \frac{1}{2}, \quad \frac{1}{2}\right)$ & $\left(\frac{1}{3}, \quad \frac{1}{3}, \quad \frac{1}{3}\right)$ & $\left(\frac{1}{4}, \quad \frac{1}{4}, \quad \frac{1}{4}\right)$ & $\left(\frac{1}{3}\right.$ \\
\hline 2 & $Q^{2} Q^{3}$ & $\left(0, \frac{1}{2}, \frac{1}{2}\right)$ & $\left(0, \frac{1}{2}, \quad \frac{1}{2}\right)$ & $\left.\frac{1}{4}, \quad \frac{1}{4}\right)$ & $\left(\frac{1}{2}\right.$ \\
\hline 3 & $-Q^{2} Q^{3}$ & $\left(\begin{array}{lll}\frac{1}{2}, & \frac{1}{2}, & \frac{1}{2}\end{array}\right)$ & $\left(\frac{1}{3}, \quad \frac{1}{3}, \quad \frac{1}{3}\right)$ & $\left(\frac{1}{4}, \quad \frac{1}{4}, \quad \frac{1}{4}\right)$ & $\left(\frac{1}{3}, \quad \frac{1}{3}\right.$ \\
\hline 4 & $Q^{2}=Q^{3}$ & $\left(\frac{1}{2}, 1, \quad \frac{1}{2}\right)$ & $\left(\frac{1}{4}, \quad \frac{1}{2}, \quad \frac{1}{4}\right)$ & $\left(\frac{1}{4}, \quad 0, \frac{1}{4}\right)$ & $\left(\begin{array}{lll}\frac{1}{2}, & 0, & \frac{1}{2}\end{array}\right)$ \\
\hline 5 & $Q^{2} Q^{3}$ & $(0,1,1)$ & $\left(0, \frac{1}{2}, \frac{1}{2}\right)$ & $\left(\frac{1}{2}, \quad 0, \quad 0\right)$ & $\left(\begin{array}{lll}1, & 0, & 0\end{array}\right)$ \\
\hline 6 & $Q^{1} Q^{2} Q^{3}$ & $\left(\begin{array}{lll}0, & 0, & 0\end{array}\right)$ & $\left(\begin{array}{lll}0, & 0, & 0\end{array}\right)$ & $\left(\frac{1}{2}, \quad \frac{1}{2}, \quad \frac{1}{2}\right)$ & $\left(\frac{1}{3}, \quad \frac{1}{3}\right.$, \\
\hline
\end{tabular}

Table 1: Non normalized $\overline{\boldsymbol{\psi}}$, and normalized $\psi$ versions of $\beta$ index when $m=1$.

\subsection{Example of non symmetric 3-voters voting games}

In this sub-part, our two considered spatial indices should be different. We first begin by an example, make a comparison and thereafter, we will give some interpretations based on various ideologies.

\subsubsection{A veto power player game}

This case is an example of non symmetric game, to show the difference between our two spatial indices since the two sets $\left\{\mathcal{D}_{i}, i \in N\right\}$ and $\left\{\mathcal{M}_{i}, i \in N\right\}$ are no longer the same. In 
fact, let us define this voting game such as voter 1 is the veto player. Formally, the game is given by $\mathcal{M}=\{12,13\}$ (corresponding to the weighted game $[3 ; 2,1,1]$ ). It is clear that, voters 2 and 3 being symmetric, by $j, k=2,3 ; k \neq j$, we have from formulae (3D) and $(3 \mathrm{~F})$,

$$
\Psi_{1}^{B}(\xi):=p_{\xi}^{j} \times\left(1-p_{\xi}^{k}\right)+p_{\xi}^{k} \times\left(1-p_{\xi}^{j}\right)+p_{\xi}^{j} \times p_{\xi}^{k}, \quad \text { and } \quad \Psi_{j}^{B}(\xi):=p_{\xi}^{1} \times\left(1-p_{\xi}^{k}\right)
$$

Whereas,

$$
\Psi_{1}^{P G}(\xi):=p_{\xi}^{j} \times\left(1-p_{\xi}^{k}\right)+p_{\xi}^{k} \times\left(1-p_{\xi}^{j}\right), \quad \text { and } \quad \Psi_{j}^{P G}(\xi):=p_{\xi}^{1} \times\left(1-p_{\xi}^{k}\right)
$$

From the two previous examples, we can infer the following results.

In dimension 1 (i.e. $m=1$ ): from Claim 1, we have,

- The non normalized Banzhaf spatial index is,

$$
\overline{\boldsymbol{\psi}}^{\boldsymbol{B}}:=\left(\bar{\psi}_{1}^{B}, \bar{\psi}_{2}^{B}, \bar{\psi}_{3}^{B}\right)=\left(\frac{3}{4}-Q^{2} Q^{3}, \frac{1}{4}-Q^{1} Q^{3}, \frac{1}{4}-Q^{1} Q^{2}\right) .
$$

- The non normalized Public Good spatial index is,

$$
\overline{\boldsymbol{\psi}}^{P \boldsymbol{P}}:=\left(\bar{\psi}_{1}^{P G}, \bar{\psi}_{2}^{P G}, \bar{\psi}_{3}^{P G}\right)=\left(\frac{1}{2}-2 Q^{2} Q^{3}, \frac{1}{4}-Q^{1} Q^{3}, \frac{1}{4}-Q^{1} Q^{2}\right) .
$$

In dimension 2 (i.e. $m=2$ ): from Claim 2 ,

- The non normalized Banzhaf spatial index is,

$$
\overline{\boldsymbol{\psi}}^{\boldsymbol{B}}=\left(\frac{3}{4}-\frac{1}{2}\left(Q_{1}^{2} Q_{1}^{3}+Q_{2}^{2} Q_{2}^{3}\right), \frac{1}{4}-\frac{1}{2}\left(Q_{1}^{1} Q_{1}^{3}+Q_{2}^{1} Q_{2}^{3}\right), \frac{1}{4}-\frac{1}{2}\left(Q_{1}^{1} Q_{1}^{2}+Q_{2}^{1} Q_{2}^{2}\right)\right) .
$$

- The non normalized Public Good spatial index is,

$$
\overline{\boldsymbol{\psi}}^{\boldsymbol{P G}}=\left(\frac{1}{2}-\left(Q_{1}^{2} Q_{1}^{3}+Q_{2}^{2} Q_{2}^{3}\right), \frac{1}{4}-\frac{1}{2}\left(Q_{1}^{1} Q_{1}^{3}+Q_{2}^{1} Q_{2}^{3}\right), \frac{1}{4}-\frac{1}{2}\left(Q_{1}^{1} Q_{1}^{2}+Q_{2}^{1} Q_{2}^{2}\right)\right)
$$

\subsubsection{Some qualitative results and comparisons}

Here in the non symmetric games, our two considered spatial indices as we have seen, are no longer the same. Thus, we can make some comparisons based on their ideological positions. 
In dimension 1. Considering our 3-voters market game, we usually have many differences depending on the form of constellations as shown in table 2 .

In dimension 2. We can make similar comparisons by considering various constellations as shown in table 3 .

\begin{tabular}{|c|c|c|c|}
\hline$\#$ & $\begin{array}{c}\text { Various } \\
\text { Constellations } \\
\end{array}$ & $\begin{array}{c}\text { Normalized Banzhaf } \\
\text { spatial index }\left(\psi^{B}\right)\end{array}$ & $\begin{array}{c}\text { Public Good } \\
\text { spatial index }\left(\psi^{P G}\right)\end{array}$ \\
\hline 1 & $Q^{1} Q^{2} Q^{3}$ & $(3 / 5, \quad 1 / 5, \quad 1 / 5)$ & $(1 / 2, \quad 1 / 4, \quad 1 / 4)$ \\
\hline 2 & - $Q^{2} Q^{3}$ & $(1 / 2, \quad 1 / 4, \quad 1 / 4)$ & $(0, \quad 1 / 2, \quad 1 / 2)$ \\
\hline 3 & $-\Omega^{2} \Omega^{3}$ & $(3 / 5, \quad 1 / 5, \quad 1 / 5)$ & $(1 / 2, \quad 1 / 4, \quad 1 / 4)$ \\
\hline 4 & $Q^{2}$ & $(1 / 2, \quad 1 / 3, \quad 1 / 6)$ & $(2 / 5, \quad 2 / 5, \quad 1 / 5)$ \\
\hline 5 & $Q^{2} Q^{3}$ & $(1 / 3, \quad 1 / 3, \quad 1 / 3)$ & $(0, \quad 1 / 2, \quad 1 / 2)$ \\
\hline 6 & $Q^{1} Q^{2} Q$ & $(1, \quad 0, \quad 0)$ & $(0$ \\
\hline 7 & $Q^{2} \longrightarrow Q^{1}=$ & $(2 / 3, \quad 1 / 6, \quad 1 / 6)$ & $(2 / 3, \quad 1 / 6, \quad 1 / 6)$ \\
\hline 8 & $Q^{1} Q^{2}$ & $(2 / 3, \quad 1 / 3, \quad 0)$ & $(2 / 3, \quad 1 / 3, \quad 0)$ \\
\hline
\end{tabular}

Table 2: Three veto players $V_{3}(1)$ in dimension $m=1$.

\section{Concluding remarks}

This last section is devoted to recaps, remarks and perspectives. Firstly, we have provided a new spatial power index namely the Public Good spatial index, and we have compared it to the only one existing in our model notably the Banzhaf spatial index proposed by Shenoy (1982). These two main spatial power indices have been deeply analyzed, and we have observed many similarities among both, but also some divergences depending on whether the voting rule is symmetric or not. To the best of our knowledge, there are no significant studies on this spatial approach since Shenoy (1982) proposed it. We have therefore deepened our investigations on it, and provided among others, ways of computing our both spatial power indices, especially in lower dimensions. 
Secondly, in our spatial model the fundamental assumption is the independence behaviors assumption, allowing voters to freely choose their own ideal points in the ideological space, consequently and in contrast to some existing spatial models like Shapley (1977), gives to voters the right to have same ideal points (which oftentimes occurs in real political situations). Among well-known indices with respect to such probabilistic interpretations in the literature, we can just mention so far two power indices following the considered spatial model - the Banzhaf index and the Public Good index. Even if in some specific conditions the obtention of other indices like Shapley Shubik index or the PGH index might be possible, there are not consistent with the model since they do not allow voters' independence behaviors.

Lastly, as shown by Rapoport and Golan $(1985)^{13}$, in some real case studies the Banzhaf spatial power index performs better than the most studied spatial index - the OwenShapley index. This latter observation emphasizes the great interest in our spatial model for further research. On the other hand, one might check that, the two main spatial indices in this paper satisfy the standard Dummy property, and are invariant via any rotation of center $O$ on voters' ideal points. This leads us to possible axiomatic characterizations that will be further investigated.

\section{Acknowledgment}

We are most grateful to Mathieu Martin, André de Palma and Zéphirin Ngameni for valuable suggestions. This research was supported by CY-Cergy-Paris Université from their CY-Initiative project (ANR16-IDEX-0008) and, also by Labex MME-DII (ANR11LBX-0023-01).

\footnotetext{
${ }^{13}$ Their analyses was illustrated in the case of the Israeli Knesset.
} 


\begin{tabular}{|c|c|c|c|c|c|c|c|c|c|}
\hline \multirow{2}{*}{$\#$} & \multicolumn{3}{|c|}{ Various constellations } & \multirow{2}{*}{\multicolumn{3}{|c|}{$\begin{array}{c}\text { Normalized Banzhaf } \\
\text { spatial index }\left(\psi^{B}\right)\end{array}$}} & \multirow{2}{*}{\multicolumn{3}{|c|}{$\begin{array}{c}\text { Public Good } \\
\text { spatial index }\left(\psi^{P G}\right)\end{array}$}} \\
\hline & $Q^{1}$ & $Q^{2}$ & $Q^{3}$ & & & & & & \\
\hline 1 & $(0 ; 0)$ & $(0 ; 0)$ & $(0 ; 0)$ & $(3 / 5$ & $1 / 5$ & $1 / 5)$ & $(1 / 2$ & $1 / 4$ & $1 / 4)$ \\
\hline 2 & $(0 ; 0)$ & $(0 ; 0)$ & $(1 / 2 ; 0)$ & $(3 / 5$, & $1 / 5$, & $1 / 5)$ & $(1 / 2$ & $1 / 4$ & $1 / 4)$ \\
\hline 3 & $(0 ; 0)$ & $(1 / 2 ; 0)$ & $(0 ; 1 / 2)$ & $(3 / 5$, & $1 / 5$ & $1 / 5)$ & $(1 / 2$ & $1 / 4$ & $1 / 4)$ \\
\hline 4 & $(0 ; 0)$ & $(1 / 2 ; 0)$ & $(1 / 2 ; 0)$ & $(5 / 9$, & $2 / 9$ & $2 / 9)$ & $(1 / 3$ & $1 / 3$ & $1 / 3)$ \\
\hline 5 & $(0 ; 0)$ & $(1 / 2 ; 0)$ & $(-1 / 2 ; 0)$ & $(7 / 11$ & $2 / 11$, & 2/11) & $(3 / 5$ & $1 / 5$ & $1 / 5)$ \\
\hline 6 & $(-1 / 2 ; 0)$ & $(0 ; 0)$ & $(0 ; 0)$ & $(3 / 5$, & $1 / 5$ & $1 / 5)$ & $(1 / 2$ & $1 / 4$ & $1 / 4)$ \\
\hline 7 & $(-1 / 2 ; 0)$ & $(0 ; 0)$ & $(0 ; 1 / 2)$ & $(3 / 5$, & $1 / 5$ & $1 / 5)$ & $(1 / 2$ & $1 / 4$ & $1 / 4)$ \\
\hline 8 & $(-1 / 2 ; 0)$ & $(0 ; 0)$ & $(1 / 2 ; 0)$ & (6/11, & $3 / 11$, & $2 / 11)$ & $(4 / 9$ & $3 / 9$ & $2 / 9)$ \\
\hline 9 & $(-1 / 2 ; 0)$ & $(0 ; 0)$ & $(-1 / 2 ; 0)$ & $(6 / 9$, & $2 / 9$ & $1 / 9)$ & $(4 / 7$ & $1 / 7$ & $2 / 7)$ \\
\hline 10 & $(-1 / 2 ; 0)$ & $(0 ; 1 / 2)$ & $(0 ; 1 / 2)$ & $(5 / 9$, & $2 / 9$ & $2 / 9)$ & $(1 / 3$ & $1 / 3$ & $1 / 3)$ \\
\hline 11 & $(-1 / 2 ; 0)$ & $(0 ; 1 / 2)$ & $(-1 / 2 ; 0)$ & $(3 / 9$, & $1 / 9$, & $2 / 9)$ & $(4 / 7$ & $1 / 7$ & $2 / 7)$ \\
\hline 12 & $(-1 / 2 ; 0)$ & $(0 ;-1 / 2)$ & $(0 ; 1 / 2)$ & $(7 / 11$ & $2 / 11$, & $2 / 11)$ & $(3 / 5$ & $1 / 5$ & $1 / 5)$ \\
\hline 13 & $(-1 / 2 ; 0)$ & $(1 / 2 ; 0)$ & $(0 ; 1 / 2)$ & $(6 / 11$, & $2 / 11$, & $3 / 11)$ & $(4 / 9$ & $2 / 9$ & $3 / 9)$ \\
\hline 14 & $(-1 / 2 ; 0)$ & $(1 / 2 ; 0)$ & $(1 / 2 ; 0)$ & $(5 / 11$ & $3 / 11$, & $3 / 11)$ & $(1 / 4$ & $3 / 8$ & $3 / 8)$ \\
\hline 15 & $(-1 / 2 ; 0)$ & $(1 / 2 ; 0)$ & $(-1 / 2 ; 0)$ & $(7 / 11$ & $1 / 11$, & $3 / 11)$ & $(6 / 10$, & 1/10, & $3 / 10)$ \\
\hline 16 & $(-1 / 2 ; 0)$ & $(-1 / 2 ; 0)$ & $(-1 / 2 ; 0)$ & $(5 / 7$, & $1 / 7$, & $1 / 7)$ & $(1 / 2$ & $1 / 4$ & $1 / 4)$ \\
\hline
\end{tabular}

Table 3: Three veto players $V_{3}(1)$ in dimension $m=2$. 


\section{Appendix}

\section{Non concentration of powers for Banzhaf spatial and Public Good indices.}

Let $V:=(N, \mathcal{W}, C(N))$ be a spatial game, and consider a sequence $\left(V_{k}\right)_{k \in \mathbb{N}}$ of spatial games with $V_{k}:=\left(N, \mathcal{W}, C_{k}(N)\right)$ such that constellations $C_{k}(N)$ tend to the constellation $C(N)$ as $k$ grows to infinity. Then any spatial power index $\boldsymbol{\psi}$ verifies the spatial continuity property if

$$
\lim _{k \rightarrow+\infty} \boldsymbol{\psi}\left(V_{k}\right)=\boldsymbol{\psi}(V)
$$

The next proposition highlights the non concentration of our two spatial indices, pushing forwards another interest of their use comparing to some existing spatial indices such as Owen-Shapley index defined in Shapley (1977). Actually, Shapley (1977) follows "ordinal approaches" of political profiles on any political issue, whereas our spatial model is rather based on "cardinal approaches".

Proposition 6 The Banzhaf and Public Good spatial indices satisfy the spatial continuity property.

Proof: straightforward, since polynomial functions are continuous. Therefore, by $2 B$, $(3 D)$ and $(3 D)$ we obtain the desired result.

\section{References}

Alonso-Meijide, J. M., Fiestras-Janeiro, M. G., García-Jurado, I., 2011. A new power index for spatial games. In: Modern Mathematical Tools and Techniques in Capturing Complexity. Springer, pp. 275285.

Banzhaf III, J. F., 1964. Weighted voting doesn't work: A mathematical analysis. Rutgers L. Rev. 19, 317.

Barr, J., Passarelli, F., 2009. Who has the power in the eu? Mathematical social sciences 57 (3), 339-366.

Benati, S., Marzetti, G. V., 2013. Probabilistic spatial power indexes. Social Choice and Welfare 40 (2), 391-410.

Brams, S. J., Lucas, W. F., Straffin Jr, P. D., 1983. Political and related models. 
Brueckner, M., 2001. Extended probabilistic characterization of power indices. In: Power Indices and Coalition Formation. Springer, pp. 29-43.

Coleman, J. S., 1971. Control of collectivities and the power of a collectivity to act. Social choice, 269-300.

Grech, P., 2019. A posteriori power indices: Organizing theory, a new index, and brexit. Available at SSRN 3353094.

Grech, P. D., 2020. Power in the council of the eu: organizing theory, a new index, and brexit. Social Choice and Welfare, 1-36.

Harman, H. H., 1976. Modern factor analysis. University of Chicago press.

Holler, M. J., 1982. Forming coalitions and measuring voting power. Political studies 30 (2), $262-271$.

Leech, D., 1990. Power indices and probabilistic voting assumptions. Public Choice 66 (3), $293-299$.

Mielcová, E., 2016. Spatial power indices with applications on real voting data from the chamber of deputies of the czech parliament. Central European Journal of Operations Research 24 (2), 407-420.

Owen, G., 1971. Political games. Naval research logistics quarterly 18 (3), 345-355.

Passarelli, F., Barr, J., 2007. Preferences, the agenda setter, and the distribution of power in the eu. Social Choice and Welfare 28 (1), 41-60.

Rapoport, A., Golan, E., 1985. Assessment of political power in the israeli knesset. American Political Science Review 79 (3), 673-692.

Shapley, L. S., 1977. A comparison of power indices and a nonsymmetric generalization.

Shapley, L. S., Shubik, M., 1954. A method for evaluating the distribution of power in a committee system. American political science review 48 (3), 787-792.

Shenoy, P. P., 1982. The banzhaf power index for political games. Mathematical Social Sciences 2 (3), 299-315.

Straffin, P. D., 1977. Homogeneity, independence, and power indices. Public Choice, 107-118.

Von Neumann, J., Morgenstern, O., 1947. Theory of games and economic behavior, 2nd rev. 